\title{
A topology-sensitive microfluidic filter for polymers of varying stiffness
}

\author{
Lisa B. Weiss, ${ }^{* \dagger}{ }^{\dagger}$ Arash Nikoubashman, ${ }^{\ddagger}$ and Christos N. Likos ${ }^{\dagger}$ \\ $\dagger$ Faculty of Physics, University of Vienna, Boltzmanngasse 5, A-1090 Vienna, Austria \\ $\ddagger$ Institute of Physics, Johannes Gutenberg University Mainz, Staudingerweg 7, 55128 \\ Mainz, Germany \\ E-mail: lisa.weiss@univie.ac.at
}

\begin{abstract}
The separation of polymers based on their size, rigidity, and topology is an essential but also highly challenging task for nanoscience and engineering. Using hybrid Molecular Dynamics simulations that correctly take into account hydrodynamics, we have designed microfluidic channels for separating linear from ring polymers in dilute solutions. We establish that the transport velocity of the polymers is independent of their topology and rigidity when the channel walls are smooth and repulsive. However, when the walls are decorated with attractive spots arranged on lines parallel to the flow, ring polymers exhibit an order of magnitude higher transport velocity compared to linear chains. The spots induce a homeotropic-like reorientation of ring polymers close to walls leading to a tank-treading motion along them, whereas linear chains are immobilized upon adsorption. This mechanism becomes more enhanced with increasing polymer rigidity. The presented technique holds thus promise for reliably separating nanoparticles based on their topology.
\end{abstract}


Biological molecules can feature varying topologies, for example DNA and RNA, which can occur in both linear and circular form. ${ }^{1-3}$ The lack of ends in circular RNA dramatically improves their resilience against degradation. ${ }^{1,4,5}$ Nature also employs highly knotted structures that are assumed to be of high relevance for the global arrangement of DNA, ${ }^{6-8}$ whereas synthetic chemists have been developing techniques to construct macromolecules of controlled topology and knotedness. ${ }^{9,10}$ Polymer topology also plays a crucial role in technological applications, as it can influence the rheological properties of polymer melts: the viscosity of ring polymer melts is drastically changed by the presence of linear contaminants. ${ }^{11}$ To exploit topology and the resulting distinct behavior and properties for novel materials, scientists are in need of reliable separation strategies. ${ }^{2,11-13}$ Currently, liquid chromatography at the critical condition or gel electrophoresis are frequently used depending on the desired topological separation. ${ }^{8,14-16}$ While the former method suffers from impurities, ${ }^{11}$ the latter is restricted to charged polymers. ${ }^{17}$

In principle, nano- and microfluidic devices can overcome these hurdles, and allow for an automated separation of the molecules in a continuous way. ${ }^{18}$ Flow-induced separation techniques are independent of the specific polymer chemistry, rendering them more widely applicable compared to electrostatic methods such as gel-electrophoresis. For instance, previous strategies used channels of varying cross-section to separate (uncharged) polymers based on their shape and size. ${ }^{19-21}$ Moreover, microfluidic devices are not only limited to the use in polymer science, but are highly versatile: ${ }^{22}$ they have proven themselves reliable for sorting cells in channels decorated with pillars, ${ }^{23,24}$ for focusing rigid particles by using inertia in Newtonian liquids ${ }^{25-27}$ or viscoelastic effects in polymer solutions. ${ }^{28-30}$ Nature exploits as well the focusing of soft, deformable vesicles in the center of blood vessels. ${ }^{31,32}$ In addition, these "labs on a chip" are promising candidates for single, un-amplified and un-chopped polymer analysis, ${ }^{33,34}$ an essential step for understanding the global spatial arrangement of DNA, which is crucial for transcription in cells. ${ }^{35}$ Finally, single genome analysis gives valuable insights in genetic mosaicism responsible for tumor formation. ${ }^{36}$ Besides separation, 
hydrodynamics can be as well used to efficiently trap particles. ${ }^{37,38}$ In general, however, the interplay between ring topology and hydrodynamics is not sufficiently well explained and understood. ${ }^{39}$

We propose a microfluidic device with a slit-like geometry, where the channel walls are decorated with tracks of attractive spots. In this setup, linear polymers adsorb on the spots and are immobilized, whereas rings are transported though being (partly) adsorbed, allowing for a truly continuous separation of the two species. In the case of ring polymers, the attractive spots enforce a tank-treading motion as the dominant dynamical pattern, enabling transport with a finite-velocity, in contrast to chains that become immobilized on the spots. Further, this tank-treading motion is accompanied by a change in orientation from planar to homeotropic. To recover the adsorbed chains, the device can be flushed by a poor solvent resulting in the collapse and evacuation of the remaining adsorbed polymers.

Pressure-driven flow of a Newtonian liquid in slit-like channels leads to a parabolic solvent velocity profile, where the shear gradient varies linearly in confinement (z)-direction. A measure for the shear experienced by a polymer is the dimensionless Weissenberg number $\mathrm{Wi}=\dot{\gamma} \tau_{0}, \dot{\gamma}$ being the shear rate and $\tau_{0}$ the polymer's longest relaxation time (see SI for details). Polymers under shear show a variety of conformations and motions that cannot be captured by techniques measuring averaged quantities. ${ }^{40}$ To get microscopic insights of polymer conformations, fluorescence microscopy is often employed in experiments as in Refs. 41-43. However, such measurements, are limited by the time- and spatial resolution of the microscope, as well as the availability of appropriate staining agents, since the vast majority of polymers is non-fluorescent. Further, it is still not clear whether staining has a significant impact on material properties or not. ${ }^{44,45}$ In the past decade, computer simulations have become invaluable for studying the flow properties of polymers, as they can provide a detailed nanoscale picture. ${ }^{46-50}$ Furthermore, simulations allow for an efficient and systematic exploration of parameter space.

This work aims at elucidating the effect of polymer topology and rigidity on the polymers' 
transport properties. We chose a generic bead-spring polymer model to reveal the principal mechanisms, instead of concentrating on a single specific monomer chemistry. A bending potential was employed to simulate polymers of varying stiffness, modifying their persistence length $L_{\mathrm{P}}$, which we express in multiples of the contour length $L_{\mathrm{C}}$. All simulations were conducted at dilute polymer concentrations. Further simulation details, in particular the inclusion of hydrodynamic interactions via an explicit solvent using the Multi Particle Collision algorithm, ${ }^{51,52}$ can be found in the Supporting Information.

We first investigated the transport and migration behavior of linear and ring polymers for varying flow strengths and rigidities in a bare slit channel with purely smooth, repulsive walls. This channel geometry has been chosen because it is easy to fabricate and commonly used in experimental setups. ${ }^{53}$ The cross-streamline migration of linear polymers was investigated experimentally, ${ }^{54}$ theoretically ${ }^{50,55}$ and as well computationally. ${ }^{49}$ Here, a repulsive hydrodynamic interaction between polymers and the channel walls was identified, as well as a movement away from the channel center due to the anisotropic diffusivity caused by the chain's deformation. However, the migration behavior of ring polymers in pressure driven flow has not been investigated yet.

Li et al. showed in recent experiments using elongational flow that the stretching of linear polymers sets in at smaller Weissenberg numbers compared to ring polymers, ${ }^{58}$ the reasons for this distinct behaviour were recently investigated. ${ }^{39}$ Since in Poiseuille flow polymers experience a broad range of shear rates, we tested the possibility to separate them by exploiting their distinct sensitivity to shear. We have tested two channel widths corresponding to 4 and 8 times the radius of gyration $R_{\mathrm{g}}$ of a linear flexible chain $\left(R_{g}=4.17 \pm 0.03 a_{0}\right.$ for a chain of 40 monomers) and varied the flow strength. (Note that the $R_{g}$ of a flexible ring is smaller for the same number of monomers $N=40$, i.e. $R_{g}=3.1 \pm 0.01 a_{0}$.) We found that ring polymers show a variety of motions and conformations; amongst them tumbling and tank-treading as previously observed in standard Couette flow. ${ }^{56,57}$ In such bare channels we observed only minor differences $(<5 \%)$ in the relative transport velocities between chains 


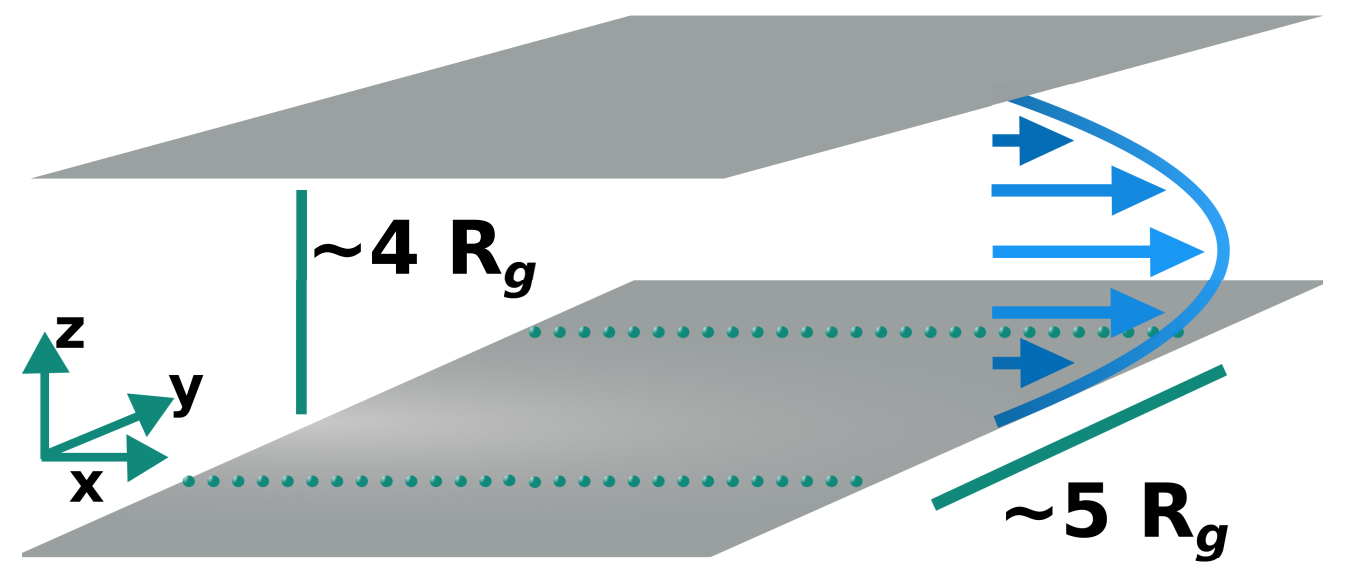

Figure 1: Scheme of a channel decorated with attractive spots, indicating the flow $(x)$, vorticity $(y)$ and gradient $(z)$ direction. Distances are indicated in multiples of $R_{\mathrm{g}}$ of a linear flexible chain.

and rings of the same molecular weight, making an efficient separation of the two species difficult (see SI for details). Therefore, more elaborate channels are needed for topological filtering.

Recently, Marenda et al. employed computer simulations without hydrodynamics and observed differences in the diffusivity of ring polymers of varying knot complexity in topograhpically modulated channels. ${ }^{59}$ Here, we present an alternative strategy by decorating the channel walls with attractive spots in addition to a short-ranged, purely repulsive wall potential. Previous simulations have demonstrated that this approach can be indeed used to separate dendrimers from linear polymers. ${ }^{60}$ In principle, such spots could be experimentally realized by E-beam lithography, thermal scanning probe lithography, or adsorption of polymers onto the substrate. ${ }^{61,62}$ In addition to the purely repulsive wall potential, the point-like surface spots exhibit an attraction with the monomers via

$$
U_{\mathrm{spot}}=-\varepsilon\left(\frac{\sigma}{r}\right)^{6}
$$

with $r$ being the distance between a monomer and a spot. This potential represents the attractive part of the van-der-Waals potential, i.e. a common interaction that does not demand a specific chemistry. The repulsive wall potential prevents monomers of penetrating 

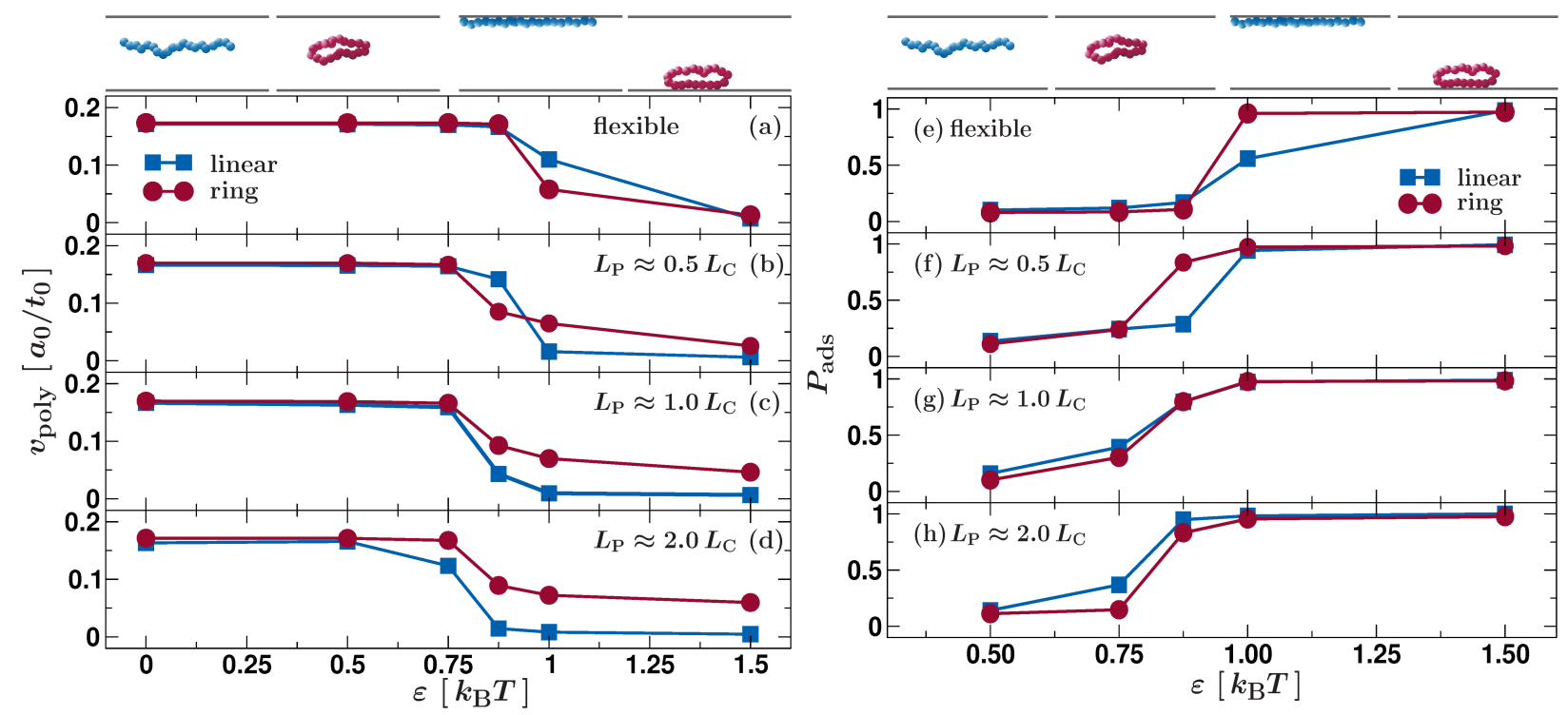

Figure 2: (a)-(d): The average transport velocity $v_{\text {poly }}$ as a function of the spots' attraction strength $\varepsilon$. The persistence length $L_{\mathrm{P}}$ in units of $L_{\mathrm{C}}$ is increasing from panel (a)-(d), linear polymers are in blue, ring polymers in red. (e)-(h): Probability $P_{\text {ads }}$ that a polymer is adsorbed with at least one monomer as a function of the attraction strength $\varepsilon$. From panel (e)-(h) the persistence length $L_{\mathrm{P}}$ in units of $L_{\mathrm{C}}$ is increasing. In all panels error bars are indicated or smaller than the symbol size. The schemes indicate the regimes where the polymers are flowing freely or are adsorbed to the channel walls.

the channel walls. We have also tested an alternative implementation, where the spots have excluded volume and are protruding from the walls, but did not find any sizable impact on the resulting polymer behavior (see SI for details). Since the interaction potential is shortranged, the polymers can only be adsorbed once they diffused close enough to the walls. Thus, narrow channels are preferable for reducing the necessary diffusion time.

To exploit the linear topology of chains, the attractive spots were aligned on a track. To increase the probability of adsorbing a polymer, each channel wall was equipped with multiple tracks separated by $\approx 5 R_{\mathrm{g}}$ in the vorticity direction as depicted in Fig. 1 . This distance is sufficiently large to avoid adsorption to multiple tracks, but small enough to minimize the diffusive time until polymers encounter attractive spots. The distance between consecutive spots corresponds to approximately the length of a Kuhn segment. Further, we have chosen relatively small flow strengths (polymer Reynolds number $\operatorname{Re}<1$, maximum Weissenberg number at the channel walls $\mathrm{Wi} \leq 70$ for flexible chains) to minimize the hydrodynamic repulsion from the channel walls. 
Figures 2 (a)-(d) depict the effect of attractive spots on the mean transport velocity of polymers as a function of the monomer-spot attraction strength. As expected, weakly attracting spots do not influence the polymers transport. In contrast, at the highest tested attraction strength of $\varepsilon=1.5 k_{\mathrm{B}} T$ linear polymers are firmly adsorbed to the walls, leading to an average transport velocity of $v_{\mathrm{L}} \approx 0$, whereas rings flow considerably faster. The ratio of $v_{\mathrm{R}}$ to $v_{\mathrm{L}}$ shows an impressive increase of up to an order of magnitude (see Fig. 3). Since linear chains are firmly adsorbed and do not migrate, this approach offers a promising pathway to separate polymers of distinct topologies continuously. Note that the ratio $v_{\mathrm{R}} / v_{\mathrm{L}}$ tends to infinity in that case and the finite value here is owed to a finite simulation length. Hence, even fully-flexible chains with a ratio of $v_{\mathrm{R}} / v_{\mathrm{L}} \approx 2$ can be separated, because chains are sticking to the walls.

Although in most cases $v_{\mathrm{R}} / v_{\mathrm{L}}$ increases monotonically with increasing $\varepsilon$, remarkably, for rather flexible polymers there is a cross-over in the mobility of distinct topologies. For fully flexible polymers, this cross-over occurs approximately at $\varepsilon=1.0 k_{\mathrm{B}} T$ and for polymers with a persistence length $L_{\mathrm{P}} \approx 0.5 L_{\mathrm{c}}$ approximately at $\varepsilon=0.875 k_{\mathrm{B}} T$. These findings can be rationalized by computing the probability that a polymer is adsorbed at the wall as shown in Fig. 2 (e)-(h). Here, we define a polymer as adsorbed when at least one monomer is within a spots' interaction radius $r_{c}=3 a_{0}$. Comparing panels (e) and (f) reveals that rings have a higher probability of being adsorbed at these attraction strengths. For a tight adsorption, the gain in potential energy has to compete with the loss in entropy that an adsorption entails. Consequently, more flexible polymers adsorb at higher $\varepsilon$ compared to more rigid polymers of the same topology. Moreover, flexible chains experience a higher entropic loss upon adsorption compared to their ring analogue. Therefore, the cross-over in $v_{\mathrm{R}} / v_{\mathrm{L}}$ exists only for rather flexible polymers.

These probabilities of adsorbing and desorbing are supported by analyzing the representative trajectories. Figure 4 shows a characteristic time series of the fraction $q$ of adsorbed monomers for fully flexible chains and rings at $\varepsilon=1.0 k_{\mathrm{B}} T$. At small attraction strengths, 


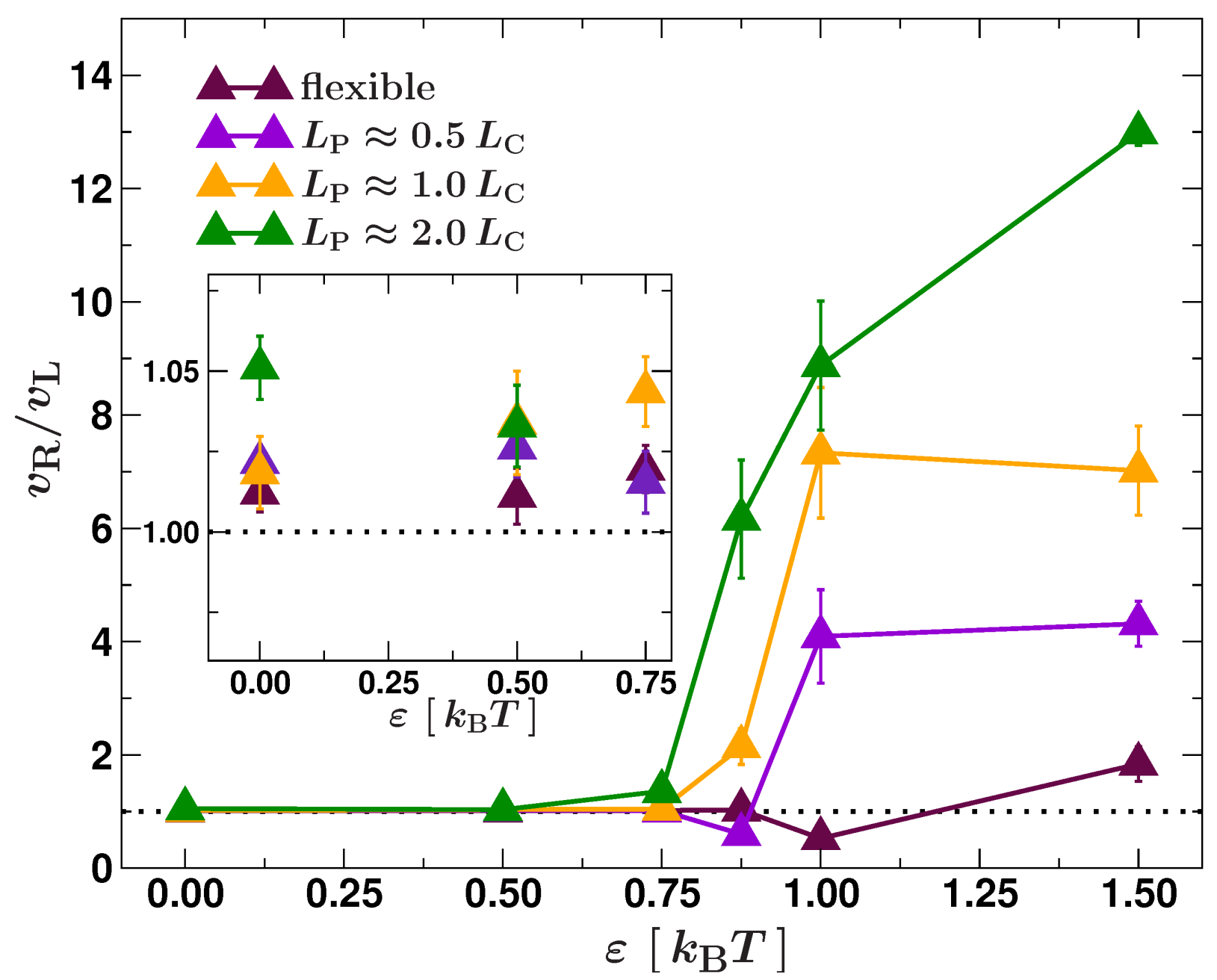

Figure 3: The relative increase of the transport velocity of ring polymers $v_{\mathrm{R}}$ to linear ones $v_{\mathrm{L}}$ is plotted as a function of the spots' attraction strength $\varepsilon$. Polymers of various persistence length $L_{\mathrm{P}}$ are shown. Error bars are either indicated or smaller than the symbols. $\varepsilon=0 k_{\mathrm{B}} T$ corresponds to an undecorated channel wall. The inset depicts the enlargement for weak attraction strengths.

only a few monomers are adsorbed (compare Figs. S2(a),S2(g) in the SI). With increasing attraction strength this fraction is steadily increasing until full adsorption is achieved (compare Figs. S2(c),S2(i) in the SI). At the cross-over, rings are adsorbed with approximately half of their monomers, whereas chains adsorb and desorb frequently. This behavior leads to a faster transport of chains than rings in this regime.

At high attraction strengths, ring polymers are transported with a finite velocity. In contrast, chains have an approximate transport velocity of zero. At first, this behavior is surprising, as rings have an equally high average probability of being adsorbed as the chain 


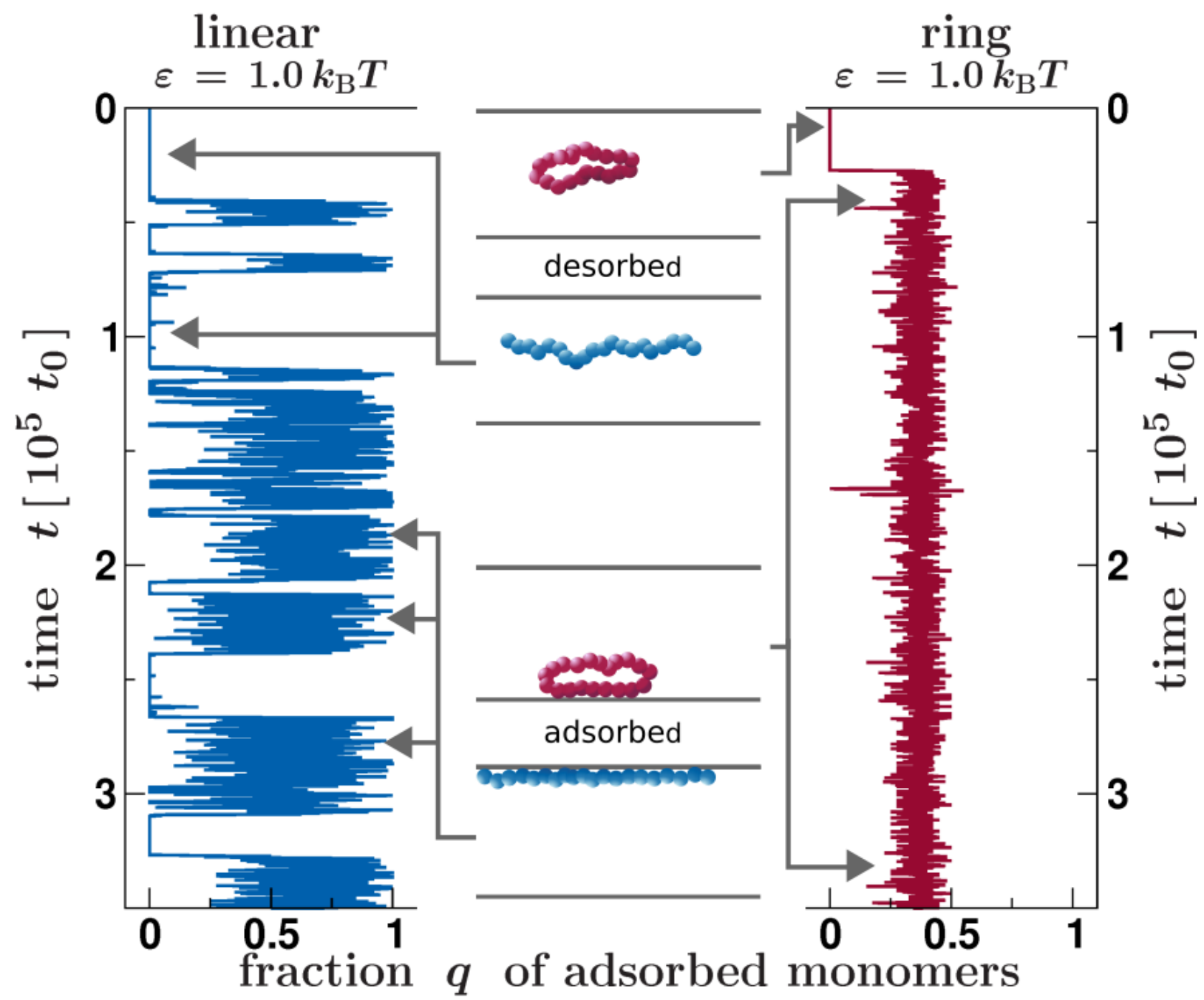

Figure 4: Representative trajectories for fully flexible chains (left, blue) and rings (right, red). The $x$-axis shows the fraction $q$ of adsorbed monomers polymers, while the $y$-axis depicts the time. The schemes help to distinguish adsorption and desorption.

polymers [Fig. 2(e)-(h)]. This purely topological effect can be explained by examining the orientation and the ensuing dynamics of ring polymers during adsorption.

In absence of attractive spots, ring polymers orient planar close to walls when they are at equilibrium. ${ }^{63}$ By applying a flow, ring polymers show a variety of dynamical motifs, including tank-treading and tumbling. To successfully tank-tread, the ring must be oriented rather homeotropic to the walls. Although this motion is present, the majority of time a ring does not perform tank-treading in the absence of attractive spots. Accordingly, the average orientation of ring polymers close to walls is planar, which was determined by a suitable 
order parameter (see details in SI). With increasing spot strength, the orientation changes from planar to homeotropic, making tank-treading the prevalent dynamical mode. A video of this tank-treading motion is available online. We emphasize that rings do not need to be fully upright to perform tank-treading, but can tilt and rear with respect to the channel wall as can be seen in the video.

Up to now, we have shown that separation of linear polymers from ring polymers can be achieved by decorating the channel walls with attractive spots. Although both polymer topologies are firmly adsorbed, ring polymers are transported significantly faster, since they perform a tank-treading motion. The open question is, how can adsorbed chains be recovered to make the filtering device functioning again. We tested stronger flows, a strategy which did not work successfully for all rigidities. Therefore, we propose an alternative strategy where the channel is flushed with a poor solvent, leading to the desorption and recovery of both species. In the simulations, the solvent quality is changed by tuning the attraction strength $\varepsilon_{\mathrm{MM}}$ in the effective monomer-monomer interaction potential, where $\varepsilon_{\mathrm{MM}}=0 k_{\mathrm{B}} T$ and $\varepsilon_{\mathrm{MM}}=1 k_{\mathrm{B}} T$ correspond to good and poor solvent conditions, respectively (see SI). Figure 5 shows representative trajectories of the effect of a solvent exchange. At $t=0$ solvent is exchanged and polymers start to desorb after some time. The worse the solvent quality, the easier it is to trigger desorption. The effect of the solvent exchange is to facilitate desorption, leading to finite transport velocity, although re-adsorption is not completely prevented, as can be seen in Fig. 5. To shorten the cleansing time, one could combine this effect with stronger flows.

In this work we have demonstrated an efficient and continuous mechanism for separating polymers depending on their stiffness and topology. As a first approach we tried to exploit the distinct migration behavior in a bare slit channel. However, the average transport velocity differed only slightly. Therefore, we propose a channel decorated with attractive spots. Our data show that linear chains adsorb firmly onto the spots reducing their transport velocity to zero. For the rings, however, the attractive spots promote tank-treading as the 


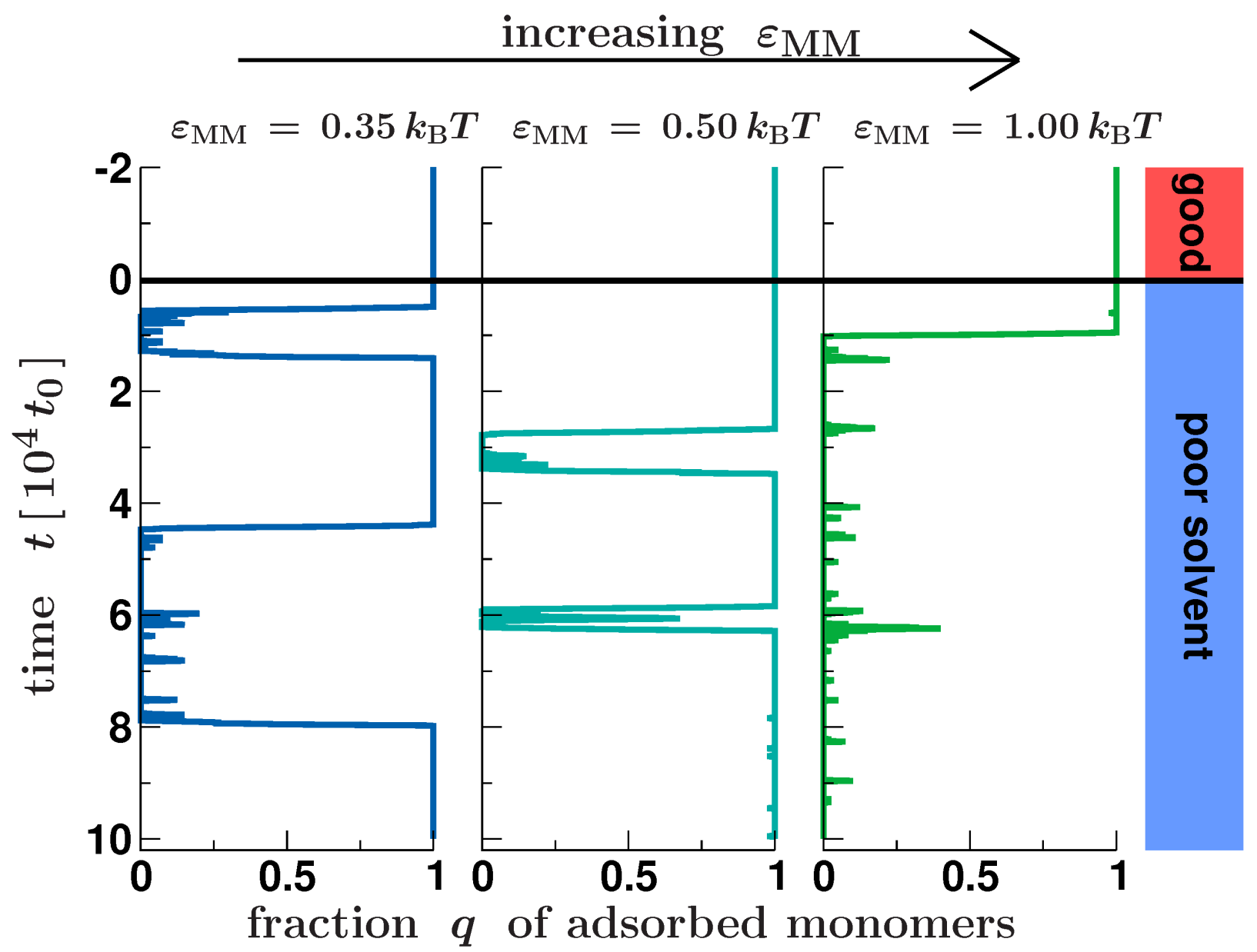

Figure 5: Representative trajectories showing the fraction $q$ of adsorbed monomers for polymers with a persistence length $L_{\mathrm{P}} \approx 1 L_{\mathrm{C}}$. The three panels clarify the effect of worsening solvent quality with increasing monomer-monomer attraction strength $\varepsilon_{\mathrm{MM}}$. The attraction strength of the spots is $\varepsilon=1.0 k_{\mathrm{B}} T$, and the flow strength is not changed. At $t=0$ the solvent is exchanged. 
dominant pattern of motion, which is accompanied by a change in orientation, from planar to homeotropic. This tank-treading motion, a mode that is not available for linear polymers, results in a higher average transport velocity of circular polymers. This mechanism holds true for polymers of varying stiffness and the major advantage of the presented strategy is that it allows for a truly continuous separation. Cleaning of the filtering device is achieved by solvent exchange. Future work will extend these studies on knotted polymers. Our findings are in principle directly transferrable to experimental realization, and we hope to motivate developments in this direction.

\section{Acknowledgement}

We thank Dimitris Vlassopoulos for critical reading of the manuscript. AN acknowledges funding through the German Research Foundation (DFG) under Grants No. NI 1487/2-1. This project has received funding from the European Union's Horizon 2020 research and innovation programme under grant agreement No 674979-NANOTRANS.

\section{Supporting Information Available}

Supporting Information is available online

This material is available free of charge via the Internet at http://pubs.acs.org/.

\section{References}

(1) Lasda, E.; Parker, R. Circular RNAs: diversity of form and function. RNA 2014, 20, 1829-1842.

(2) Jeck, W. R.; Sharpless, N. E. Detecting and characterizing circular RNAs. Nat. Biotechnol. 2008, 32, 453-461. 
(3) Campbell, N. A.; Reece, J. B.; Urry, L. A.; Cain, M. L.; Wasserman, S. A.; Minorsky, P. V.; Jackson, R. B. Campbell Biology, 10th ed.; Pearson: Boston, 2013.

(4) Dong, Y.; He, D.; Peng, Z.; Peng, W.; Shi, W.; Wang, J.; Li, B.; Zhang, C.; Duan, C. Circular RNAs in cancer: an emerging key player. J. Hematol. Oncol. 2017, 1-8.

(5) Piwecka, M.; Glažar, P.; Hernandez-Miranda, L. R.; Memczak, S.; Wolf, S. A.; RybakWolf, A.; Filipchyk, A.; Klironomos, F.; Cerda Jara, C. A.; Fenske, P.; Trimbuch, T.; Zywitza, V.; Plass, M.; Schreyer, L.; Ayoub, S.; Kocks, C.; Kühn, R.; Rosenmund, C.; Birchmeier, C.; Rajewsky, N. Loss of a mammalian circular RNA locus causes miRNA deregulation and affects brain function. Science 2017, 8526, eaam8526.

(6) Wasserman, S. A.; Cozzarelli, N. R. Biochemical Topology: Applications to DNA Recombination and Replication. Science 1986, 232, 951-960.

(7) Arsuaga, J.; Vázquez, M.; Trigueros, S.; Sumners, D. W.; Roca, J. Knotting probability of DNA molecules confined in restricted volumes: DNA knotting in phage capsids. $P$. Natl. Acad. Sci. USA 2002, 99, 5373-5377.

(8) Arsuaga, J.; Vazquez, M.; McGuirk, P.; Trigueros, S.; Sumners, D. W.; Roca, J. DNA knots reveal a chiral organization of DNA in phage capsids. P. Natl. Acad. Sci. USA 2005, 102, 9165-9169.

(9) Fielden, S. D. P.; Leigh, D. A.; Woltering, S. L. Molecular Knots. Angew. Chem., Int. Ed. 2017, DOI: 10.1002/ange.201702531.

(10) Bielawski, C. W.; Benitez, D.; Grubbs, R. H. An "Endless" Route to Cyclic Polymers. Science 2002, 297, 2041-2044.

(11) Kapnistos, M.; Lang, M.; Vlassopoulos, D.; Pyckhout-Hintzen, W.; Richter, D.; Cho, D.; Chang, T.; Rubinstein, M. Unexpected power-law stress relaxation of entangled ring polymers. Nat. Mater. 2008, 7, 997-1002. 
(12) McLeish, T. Polymers Without Beginning or End. Science 2002, 1740, 2001-2002.

(13) Yamamoto, T.; Tezuka, Y. Topological polymer chemistry: a cyclic approach toward novel polymer properties and functions. Polym. Chem. 2011, 2, 1930-1941.

(14) Lee, H. C.; Lee, H.; Lee, W.; Chang, T.; Roovers, J. Fractionation of Cyclic Polystyrene from Linear Precursor by HPLC at the Chromatographic Critical Condition. Macromolecules 2000, 33, 8119-8121.

(15) Lee, W.; Lee, H.; Lee, H. C.; Cho, D.; Chang, T.; Gorbunov, A. A.; Roovers, J. Retention Behavior of Linear and Ring Polystyrene at the Chromatographic Critical Condition. Macromolecules 2002, 35, 529-538.

(16) Takano, A.; Kushida, Y.; Aoki, K.; Masuoka, K.; Hayashida, K.; Cho, D.; Kawaguchi, D.; Matsushita, Y. HPLC Characterization of Cyclization Reaction Product Obtained by End-to-End Ring Closure Reaction of a Telechelic Polystyrene. Macromolecules 2007, 40, 679-681.

(17) Dorfman, K. D. Taylor-Aris Dispersion During Lubrication Flow in a Periodic Channel. Chem. Eng. Comm. 2009, 197, 39-50.

(18) Dorfman, K. D.; King, S. B.; Olson, D. W.; Thomas, J. D. P.; Tree, D. R. Beyond Gel Electrophoresis: Microfluidic Separations, Fluorescence Burst Analysis, and DNA Stretching. Chem. Rev. 2013, 113, 2584-2667.

(19) Han, J.; Craighead, H. G. Separation of Long DNA Molecules in a Microfabricated Entropic Trap Array. Science 2000, 288, 1026-1029.

(20) Mikkelsen, M. B.; Reisner, W.; Flyvbjerg, H.; Kristensen, A. Pressure-driven DNA in Nanogroove Arrays: Complex Dynamics Leads to Length- and Topology-Dependent Separation. Nano Lett. 2011, 11, 1598-1602. 
(21) Ollila, S. T. T.; Denniston, C.; Karttunen, M.; Ala-Nissila, T. Biopolymer Filtration in Corrugated Nanochannels. Phys. Rev. Lett. 2014, 112, 118301.

(22) Stone, H. A.; Stroock, A. D.; Ajdari, A. ENGINEERING FLOWS IN SMALL DEVICES: Microfluidics Toward a Lab-on-a-Chip. Annu. Rev. Fluid Mech. 2004, 36, 381-411.

(23) Krüger, T.; Holmes, D.; Coveney, P. V. Deformability-based red blood cell separation in deterministic lateral displacement devices - A simulation study. Biomicrofluidics 2014, 8,054114

(24) Geislinger, T. M.; Franke, T. Sorting of circulating tumor cells (MV3-melanoma) and red blood cells using non-inertial lift. Biomicrofluidics 2013, 7, 044120.

(25) Segré, G.; Silberberg, A. Radial Particle Displacement in Poiseuille Flow of Suspensions. Nature 1961, 189, 209-210.

(26) Prohm, C.; Gierlak, M.; Stark, H. Inertial microfluidics with multi-particle collision dynamics. Eur. Phys. J. E 2012, 35, 80.

(27) Laumann, M.; Bauknecht, P.; Gekle, S.; Kienle, D.; Zimmermann, W. Cross-stream migration of asymmetric particles driven by oscillating shear. EPL Europhys. Lett. $2017,117,44001$.

(28) Young Kim, J.; Won Ahn, S.; Sik Lee, S.; Min Kim, J. Lateral migration and focusing of colloidal particles and DNA molecules under viscoelastic flowSPI. Lab Chip 2012, 12, 2807.

(29) Lim, E. J.; Ober, T. J.; Edd, J. F.; Desai, S. P.; Neal, D.; Bong, K. W.; Doyle, P. S.; McKinley, G. H.; Toner, M. Inertio-elastic focusing of bioparticles in microchannels at high throughput. Nat. Commun. 2014, 5, 4120. 
(30) Howard, M. P.; Panagiotopoulos, A. Z.; Nikoubashman, A. Inertial and viscoelastic forces on rigid colloids in microfluidic channels. J. Chem. Phys. 2015, 142, 224908.

(31) Noguchi, H.; Gompper, G. Shape transitions of fluid vesicles and red blood cells in capillary flows. P. Natl. Acad. Sci. USA 2005, 102, 14159-14164.

(32) Bächer, C.; Schrack, L.; Gekle, S. Clustering of microscopic particles in constricted blood flow. Phys. Rev. Fluids 2017, 2, 013102.

(33) Reisner, W.; Pedersen, J. N.; Austin, R. H. DNA confinement in nanochannels: physics and biological applications. Rep. Prog. Phys. 2012, 75, 106601.

(34) Chiu, D. T.; DeMello, A. J.; Di Carlo, D.; Doyle, P. S.; Hansen, C.; Maceiczyk, R. M.; Wootton, R. C. Small but Perfectly Formed? Successes, Challenges, and Opportunities for Microfluidics in the Chemical and Biological Sciences. Chem 2017, 2, 201-223.

(35) Hanahan, D.; Weinberg, R. A. Hallmarks of Cancer: The Next Generation. Cell 2011, $144,646-674$.

(36) Gawad, C.; Koh, W.; Quake, S. R. Single-cell genome sequencing: current state of the science. Nat. Rev. Genet. 2016, 17, 175-188.

(37) Tanyeri, M.; Schroeder, C. M. SI Manipulation and Confinement of Single Particles Using Fluid Flow. Nano Lett. 2013, 13, 2357-2364.

(38) Shenoy, A.; Rao, C. V.; Schroeder, C. M. Stokes trap for multiplexed particle manipulation and assembly using fluidics. P. Natl. Acad. Sci. USA 2016, 113, 3976-3981.

(39) Hsiao, K.-W. W.; Schroeder, C. M.; Sing, C. E. Ring Polymer Dynamics Are Governed by a Coupling between Architecture and Hydrodynamic Interactions. Macromolecules 2016, 49, 1961-1971.

(40) LeDuc, P.; Haber, C.; Bao, G.; Wirtz, D. Dynamics of individual flexible polymers in shear flow. Letters to Nature 1999, 399, 564-566. 
(41) Smith, D. E.; Babcock, H. P.; Chu, S. Single-Polymer Dynamics in Steady Shear Flow. Science 1999, 283, 1724-1727.

(42) Doyle, P. S.; Ladoux, B.; Viovy, J.-L.; Sing, C. E.; Selvidge, J. G.; Alexander-Katz, A.; Perkins, T. T.; Smith, D. E.; Chu, S.; Milner, S. T.; Newhall, J. D.; Doyle, P. S.; Ladoux, B.; Viovy, J.-L.; Bielawski, C. W.; Benitez, D.; Grubbs, R. H.; AlexanderKatz, A.; Netz, R. R. Dynamics of a Tethered Polymer in Shear Flow. Phys. Rev. Lett. 2000, 84, 4769-4772.

(43) Schroeder, C. M.; Teixeira, R. E.; Shaqfeh, E. S. G.; Chu, S. Characteristic Periodic Motion of Polymers in Shear Flow. Phys. Rev. Lett. 2005, 95, 018301.

(44) Kundukad, B.; Yan, J.; Doyle, P. S. Effect of YOYO-1 on the mechanical properties of DNA. Soft Matter 2014, 10, 9721-9728.

(45) Shaqfeh, E. S. G. The dynamics of single-molecule DNA in flow. J. Non-Newt. Fluid Mech. 2005, 130, 1-28.

(46) Cannavacciuolo, L.; Winkler, R. G.; Gompper, G. Mesoscale simulations of polymer dynamics in microchannel flows. EPL Europhys. Lett. 2008, 83, 34007.

(47) Katkar, H. H.; Muthukumar, M. Effect of charge patterns along a solid-state nanopore on polyelectrolyte translocation. J. Chem. Phys. 2014, 140, 135102.

(48) Usta, O. B.; Butler, J. E.; Ladd, A. J. C. Transverse Migration of a Confined Polymer Driven by an External Force. Phys. Rev. Lett. 2007, 98, 098301.

(49) Jendrejack, R. M.; Schwartz, D. C.; De Pablo, J. J.; Graham, M. D. Shear-induced migration in flowing polymer solutions: Simulation of long-chain DNA in microchannels. J. Chem. Phys. 2004, 120, 2513-2529.

(50) Reddig, S.; Stark, H. Cross-streamline migration of a semiflexible polymer in a pressure driven flow. J. Chem. Phys. 2011, 135, 165101. 
(51) Malevanets, A.; Kapral, R. Continuous-velocity lattice-gas model for fluid flow. EPL Europhys. Lett. 1998, 44, 552-558.

(52) Malevanets, A.; Kapral, R. Mesoscopic model for solvent dynamics. J. Chem. Phys. 1999, 110, 8605-8613.

(53) Stein, D.; van der Heyden, F. H. J.; Koopmans, W. J. a.; Dekker, C. Pressure-driven transport of confined DNA polymers in fluidic channels. P. Natl. Acad. Sci. USA 2006, 103, 15853-15858.

(54) Steinhauser, D.; Koster, S.; Pfohl, T. Mobility Gradient Induces Cross-Streamline Migration of Semiflexible Polymers. ACS Macro Lett. 2012, 1, 541-545.

(55) Ma, H.; Graham, M. D. Theory of shear-induced migration in dilute polymer solutions near solid boundaries. Phys. Fluids 2005, 17, 083103.

(56) Lang, P. S.; Obermayer, B.; Frey, E. Dynamics of a semiflexible polymer or polymer ring in shear flow. Phys. Rev. E 2014, 89, 022606.

(57) Chen, W.; Chen, J. Z.; An, L. J. Tumbling and tank-treading dynamics of individual ring polymers in shear flow. Soft Matter 2013, 9, 4312-4318.

(58) Li, Y.; Hsiao, K.-W.; Brockman, C. a.; Yates, D. Y.; Robertson-Anderson, R. M.; Kornfield, J. a.; San Francisco, M. J.; Schroeder, C. M.; McKenna, G. B. When Ends Meet: Circular DNA Stretches Differently in Elongational Flows. Macromolecules 2015, 48, 5997-6001.

(59) Marenda, M.; Orlandini, E.; Micheletti, C. Sorting ring polymers by knot type with modulated nanochannels. Soft Matter 2017, 13, 795-802.

(60) Nikoubashman, A.; Likos, C. N. Flow-induced polymer translocation through narrow and patterned channels. J. Chem. Phys. 2010, 133, 074901. 
(61) Park, S. M.; Liang, X.; Harteneck, B. D.; Pick, T. E.; Hiroshiba, N.; Wu, Y.; Helms, B. A.; Olynick, D. L. Sub-10 nm Nanofabrication via Nanoimprint Directed Self-Assembly of Block Copolymers. ACS Nano 2011, 5, 8523-8531.

(62) Gottlieb, S.; Lorenzoni, M.; Evangelio, L.; Fernández-Regúlez, M.; Ryu, Y. K.; Rawlings, C.; Spieser, M.; Knoll, A. W.; Perez-Murano, F. Thermal scanning probe lithography for the directed self-assembly of block copolymers. Nanotechnology 2017, 28, 175301.

(63) Poier, P.; Egorov, S. A.; Likos, C. N.; Blaak, R. Concentration-induced planar-tohomeotropic anchoring transition of stiff ring polymers on hard walls. Soft Matter 2016, 12, 7983-7994. 
Graphical TOC Entry

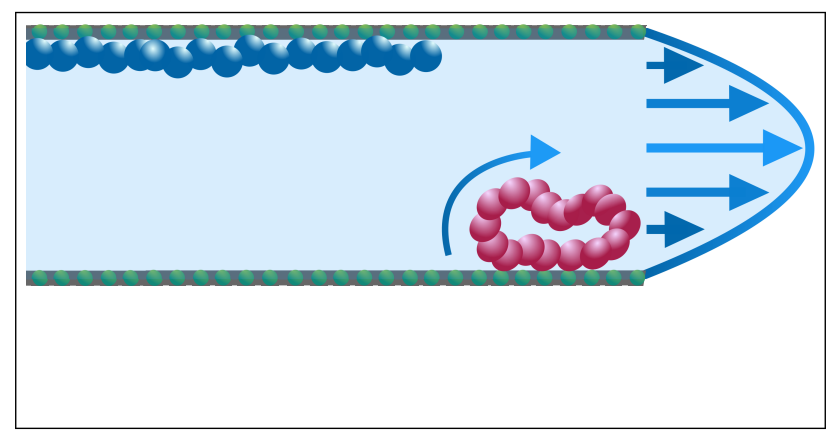

